\title{
Erratum to: Severe Mycobacterial Diseases in a Patient with GOF IKB $\alpha$ Mutation Without EDA
}

\author{
Alison Joanne Lee ${ }^{1}$ - Marcela Moncada-Vélez ${ }^{2,3}$ - Capucine Picard ${ }^{4,5,6,7}$. \\ Genevieve Llanora $^{1}$ • Chiung-Hui Huang ${ }^{1}$ - Laurent Abel ${ }^{4,5} \cdot$ Si Min Chan $^{8,9}$. \\ Bee-Wah Lee $^{1,9}$ • Jean-Laurent Casanova ${ }^{2,4,5,6,10}$ • Jacinta Bustamante J $^{2,4,5,7}$. \\ Lynette Pei-Chi Shek $^{1,9}$ • Stéphanie Boisson-Dupuis ${ }^{2,4}$
}

Published online: 18 February 2016

(C) Springer Science+Business Media New York 2016

\section{Erratum to: J Clin Immunol (2016) 36:12-15 \\ DOI 10.1007/s10875-015-0223-8}

The original version of this article unfortunately contained mistakes in Figure e where part of the immunoblot is missing. The correct version is presented below.

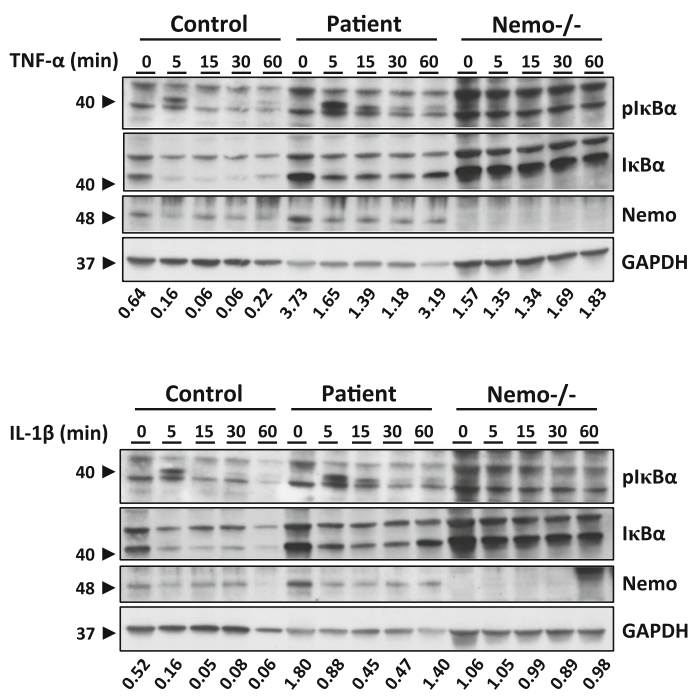

The online version of the original article can be found at http://dx.doi.org/ 10.1007/s10875-015-0223-8.

Alison Joanne Lee

alison_joanne_lee@nuhs.edu.sg

$\triangle$ Stéphanie Boisson-Dupuis

stbo603@rockefeller.edu

1 Division of Allergy and Immunology, Department of Paediatrics, Khoo Teck Puat - National University Children's Medical Institute (KTP-NUCMI), National University Hospital, 1E Kent Ridge Road, NUHS Tower Block Level 12, Singapore 119228, Singapore

2 St. Giles Laboratory of Human Genetics of Infectious Diseases, Rockefeller Branch, The Rockefeller University, 1230 York Avenue, New York, NY 10065, USA

3 Group of Primary Immunodeficiencies, Institute of Biology, University of Antioquia UdeA, Calle 70 No. 52-21,

Medellín, Colombia
4 Laboratory of Human Genetics of Infectious Diseases, Necker Branch, INSERM U1163, Necker Hospital for Sick Children, INSERM, Paris, France

5 Imagine Institute, Paris Descartes University, Paris, France

6 Pediatric Hematology-Immunology Unit, Necker Hospital for Sick Children, Paris, France

7 Center for the Study of Primary Immunodeficiencies, Necker Hospital for Sick Children, AP-HP, Paris, France

8 Division of Infectious Disease, Department of Paediatrics, Khoo Teck Puat - National University Children's Medical Institute (KTP-NUCMI), National University Hospital, Singapore, Singapore

9 Department of Pediatrics, National University of Singapore, Singapore, Singapore

10 Howard Hughes Medical Institute, New York, NY, USA 